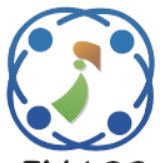

\title{
A New Method to Select the Interesting Association Rules with Multiple Criteria
}

\author{
Azzeddine Dahbi $^{1 *} \quad$ Siham Jabri $^{1} \quad$ Youssef Balouki $^{1} \quad$ Taoufiq Gadi $^{1}$ \\ ${ }^{1}$ Faculty of Science and Technology, Hassan 1st University Settat, Laboratory Informatics, \\ Imaging and Modelling of Complex Systems, Morocco \\ * Corresponding author's Email: azdine.im@gmail.com
}

\begin{abstract}
Using the association rules in datamining is one of the most relevant techniques in modern society, aiming to extract the interesting correlation and relation among sets of items or products in large transactional databases. The huge number of extracted association rules represents the main problem that a decision maker can face. Hence, the knowledge post-processing phase becomes very important and challenging to define the most interesting association rules, many interestingness measures have been proposed. Currently, there is no optimal measure that can be selected to evaluate the extracted association rules. To bypass this problem, we propose an approach based on multi-criteria optimization aiming to find a good compromise without excluding any measures. The experiments performed on numerous benchmark datasets show that the proposed algorithm is properly reducing a large number of association rules and keeping the most significant and interesting ones compared to other approaches which illustrate the efficiency and the applicability our approach.
\end{abstract}

Keywords: Datamining, Association rules, Interestingness measures, MCDA, ELECTRE method.

\section{Introduction}

Data mining, also called knowledge discovery in databases, is an important research domain in computer science, it is widely used in business (insurance, retail, banking, credit card fraud detection system), science research (medicine, astronomy, biological data analysis), and government security (detection of criminals and terrorists). One of the most important DM tasks is to find the association rules and to discover the interesting and useful patterns and relationships in large volumes of data. Currently, there are many proposed algorithms for mining association rules; the most known and simplest one is the Apriori Algorithm [1] proposed by Agrawal in 1993. The use of Apriori algorithm in Data Mining makes it possible to test the various possible combinations of the items (Data_Atributes) to find potential relationships, which will be expressed in the form of association rules. An association rule is an implication expression formed as $\mathrm{X} \rightarrow \mathrm{Y}$, where $\mathrm{X}$ and $\mathrm{Y}$ are disjoint item sets. The power of an association rule can be measured regarding its support and confidence.

The majority of existing association rules algorithms based on support and confidence produce an important number of rules. Therefore, the decision maker is incapable of finding the most useful ones and is consequently unable to make decisions. To beat this problem, diverse measures have been proposed in the literature to evaluate the interestingness of the rule [2-3].

However, the abundance of these measures existing in the literature caused a new problem, which is the selection of measures that users see the best.

Several works aim to help the user in the choice of the measure to be the most adequate to the decision scope. Some works compare the order of rules given by the interestingness measures to that yield by human experts and choose the measure that yields the nearest one to the expert ranking. However, these works were based on specific domains and datasets, and their results cannot be taken as a general conclusion as it is not always 
possible to get the expert's ranking. Other approaches and techniques were presented by studying the similarity between the measures for classifying them [5] or by proposing a set of criteria to design good interestingness measures[4]. Vaillant et al.[6] Propose to extract a pre-order on twenty measures and identify the clusters of measures. Those approaches are not guaranteed the selection of the best and the proper interestingness measures for the simple reason that this measure is not verified the used properties. Chen et al.[7] and Toloo et al. [8] propose an approach to estimate and rank the efficiency of association rules with multiple criteria using a non-parametric approach Data Envelopment Analysis (DEA) the problem here is the difficulty of the changed the fractional linear measure into a linear programming model and the complexity of the resolution. Our previous work [9] is to partition the association rules into $\mathrm{K}$ disjoint clusters and then classify the obtained clusters from the best to the worst by using an approach based on $\mathrm{k}$-means algorithm the inconvenient of this work is it can't define the number of association rules according to the user's needs, and it is not fixed and related to the number of clusters choosing. Another our previous work [10] use ELECTRE1 to select the most interesting using a set of criteria.

Our paper lies within this scope. we propose an approach based on ELECTRE method, aiming to select the association rules with high qualities which make it easy for users to choose the best ones from a huge number of association rules and using a set of criteria without privileging or excluding any measures and resolve some disadvantages of ELECTRE1 such as discarding the interesting and keeping the non-interesting ones by giving a new notion of outranking relation. An association rule R1 outranks another rule R2 not only if its measures are high than the other but we impose a predefined threshold. The credibility of our approach is demonstrated by the main advantage of the results over others which are the reduction of a large number of association rules.

The outlines of our paper is as follows: In Section 2, we present the necessary scientific background and an overview of association rules mining, interestingness measures and MCDA methods. Part 3 presents our proposed approaches based on ELECTRE method. In section 4, we discuss the experimental results and its analysis. The conclusion and scope for future work are given in the last section.

must be in English. These guidelines include complete descriptions of the fonts, spacing, and related information for producing your proceedings manuscripts.

\section{Background}

\subsection{Association rules}

Association rules present an efficient method of analysing huge binary data sets. One typical application is to discover relationships between binary variables in transaction databases, and this type of analysis is called a 'Market Basket Analysis.'

Let $I=\left\{i_{1}, i_{2}, \ldots i_{n}\right\}$ be a set of all items, association rules are generated over a large set of transactions, denoted by $T$ with $T=\left\{t_{1}, t_{2}, \ldots t_{m}\right\}$, every transaction ti is an item set and meet $t_{i} \subseteq I$.

An association rule is a statement of the form $X \rightarrow Y$, where $X, Y \subseteq I$ with the fact that $X \cap Y=\varnothing$. The set $X$ is called the antecedent of the rule, and the set $\mathrm{Y}$ is called the consequent of the rule where $\mathrm{I}$ is a non-empty set.

An association rule can be considered interesting if the items involved often occur together and there are suggestions that one of the sets might in some sense lead to the presence of the other set. The strength of an association rule can be measured by mathematical notions called: 'support,' and 'confidence.'

The notation $P(X)$ is used to represent the proportion of times that the set $X$ appears in the transaction set $T$.

The support of the rule $X \rightarrow Y$ is the percentage of transactions in a database $\mathrm{D}$ that contain $X \cup Y$ and is represented as:

$$
\operatorname{Support}(X \rightarrow Y)=P(X, Y)=\frac{n(X \cup Y)}{n}
$$

The confidence of a rule $\mathrm{X} \rightarrow \mathrm{Y}$ describes the percentage of transactions containing $X$ which also contain $\mathrm{Y}$ and is represented as

$$
\text { Confidence }(X \rightarrow Y)=\frac{P(X, Y)}{P(X)}=\frac{n(X \cup Y)}{n(X)}
$$

Where $n(X \cup Y)$ is the number of transactions that contain items (i.e., $X \cup Y$ ) of the rule $n(X)$ is the number of transactions containing itemset $X$ and $n$ is the total number of transactions.

To discover interesting association rules from the given database $D$, the support and the confidence of the rule should satisfy a user-specified support threshold called minsup and a confidence threshold called minconf. 


\subsection{Interestingness measures}

Association rules mining can generate a large quantity of rules, most of which are not interesting to the user. Interestingness measures play a significant role in data mining; they are used to find the truly interesting rules and to select and rank patterns according to their potential interest to the user. These measures can be divided into two categories: objective measures (data-driven) based on the statistical strengths or properties of the discovered rules, and subjective measures e.g. unexpectedness and action ability[11] (user driven) which are derived from the user beliefs or expectations of their particular problem domain.
Support, confidence, and lift are the most widely used objective measures to select interesting rules. In addition to these measures, there are many other objective ones introduced by Tan et al. [12], such as $\varphi$-coefficient, odds ratio, kappa, mutual information, J-measure, Gini index, Laplace, conviction, interest, and cosine. Their study shows that different measures have different intrinsic properties and classifies them from several perspectives. It also compares their properties, identifies their roles in the datamining process, and gives strategies for selecting appropriate measures for applications to come up with a conclusion that there is no measure is better than others in all applications domains.

Table 1. Some Interestingness Measures.

\begin{tabular}{|c|c|}
\hline Measures & Formula \\
\hline Lift & $\operatorname{Lift}(X \rightarrow Y)=\frac{P(X Y)}{P(X) P(Y)}$ \\
\hline Information Gain & $G I(X \rightarrow Y)=\log _{2} \frac{P(X Y)}{P(X) P(Y)}$ \\
\hline Example \& Counter Example Rate & $\operatorname{ECR}(X \rightarrow Y)=2-\frac{1}{\operatorname{conf}(X \rightarrow Y)}$ \\
\hline Jaccard & $J R D(X \rightarrow Y)=\frac{P(X Y)}{P(X \bar{Y})+P(Y)}$ \\
\hline Cosinus & $\operatorname{COS}(X \rightarrow Y)=\frac{P(X Y)}{\sqrt{P(X) P(Y)}}$ \\
\hline Pearl & $P R L(X \rightarrow Y)=P(X)|P(Y / X)-P(Y)|$ \\
\hline Loevinger & $L V G(X \rightarrow Y)=\frac{P\left(\frac{Y}{X}\right)-P(Y)}{1-P(Y)}$ \\
\hline Conviction & $C N V=\frac{P(X) P(\overline{Y)}}{P(X Y)}$ \\
\hline Zhang & $Z H N(X \rightarrow Y)=\frac{P(X Y)-P(X) P(Y)}{\max \{P(X Y) P(\bar{Y}), P(Y) P(X \bar{Y})\}}$ \\
\hline Piatetsky Shapiro & $P S(X \rightarrow Y)=P(X Y)-P(X) P(Y)$ \\
\hline Sebag-Schoenauer & $S B G(X \rightarrow Y)=\frac{P(X Y)}{P(X Y)}$ \\
\hline
\end{tabular}


Liu et al. [13] analyse the discovered association rules using the user's specifications to identify those potentially interesting ones for the user and find patterns interesting if they are unexpected (contradicting user's belief) or offer strategic information on which user can act.

Other authors [14] discover interesting rules by using a new methodology for combining data-driven (objective) and user-driven (subjective) evaluation measures. That is to say; their method revolves around the idea that the objective measures are first used to filter the rule set and then subjective criteria are used to assist the user in analysing the rules according to his/her knowledge and goals.

Razan Paul[15] uses a semantic interestingness measures for discovering association rules. Semantic interestingness measures take into account how data attributes are semantically related. It makes use of the structure of the ontology that hosts the corresponding items (e.g. generalization, specialization, etc.)

Owing to a large number of interesting measures existing in the literature, how to select suitable measures becomes a major challenge. To overcome that problem, several approaches and techniques were presented by proposing intuitive formal criteria that a good measure should verify to evaluate the degree of interest of rule [16]. Tan et al [12] discuss the properties of twenty-one measures and conclude that there is no measure better than others in all application domain.

Some objective measures are shown in Table 1 and used to evaluate the performance or interestingness of rules.

\subsection{MCDA method:}

Multi-Criteria Decision Analysis (MCDA) [17] is a general structure for supporting difficult decision-making situations with multiple and often conflicting objectives that organization groups and/or decision-makers value differently. In the last years, many MCDA methods have been improved and applied to decision problems in different domains. Among the popular research area within MCDA, there is the outranking approach and in particular ELECTRE methods [18].

Decision-making is the process of selecting a logical choice by assessing alternative occupations, defining goals, and gathering information By this way; we want not only to set as many of these propositions as possible but also to select the one that best suits with our goals, desires, objectives, and so on.
MCDA focuses on planning problems involving multiple criteria, structuring and solving decision. The goal is to support decision makers to solve problems. Ordinarily, there is the unique optimal solution for such problems, and it is important to use decision maker's preferences to differentiate between solutions.

To define a multi-attribute decision-making problem, we give $\mathrm{m}$ criteria $C_{1}, C_{2}, \ldots C_{m}$ and $\mathrm{n}$ alternatives $A_{1}, \ldots . A_{n}$. Table 2 shows a standard functionality of multi-attribute decision-making methodology. Each column describes the performance of an alternative and the rows of the table describes the criteria. The performance of alternative $A_{j}$ against criterion $C_{i}$ is described by the value $a_{i j}$. We take for granted that a higher score value means a better performance since any goal of minimization can be quickly transformed into a goal of maximization. We assign to each criteria $C_{i}$ a positive weight $w_{i}$, as shown in decision Table 2 , it reflects the relative importance of criteria $C_{i}$.

ELECTRE [18] (Elimination Et Choix Traduisant la Réalité) is one of the MCDA methods, and this method permits decision makers to select the best choice with most advantage and least conflict in the function of different criteria. We use the ELECTRE method to choose the best action from a set of other ones. Among the simplest way of ELECTRE family, we find ELECTRE1.

The goal is then to select the best alternative given the performance values of each alternative with respect to each criterion (given as an $m \times n$ decision matrix) and the corresponding weights of the criteria defined by the decision maker.

For modelling the preference information between each pair of alternatives, such as $A_{i}$ and $A_{k}$ $(i, \quad k=1,2, \ldots m)$, ELECTRE uses the concept of outranking relations. Alternative $A_{\mathrm{i}}$ outranks $A_{k}$ if on a great part of the criteria $A_{i}$ performs at least as good as $A_{k}$ (concordance condition), while its worse performance is still acceptable on the other criteria (non-discordance condition). After having determined for each pair of alternatives whether one alternative outranks another, these pairwise outranking assessments can be combined into a part or complete ranking.

The outranking method aims to find all alternatives that dominate the other ones and they cannot be dominated by any other alternatives. Each criterion is assigned a subjective weight $w_{k}$ by the decision maker, where:

$$
\sum_{i=1}^{N} w_{i}=1
$$


Table 2. Matrix decision

\begin{tabular}{|c|c|c|c|c|}
\hline & & $A 1$ & $\ldots \ldots$ & $A n$ \\
\hline $\mathrm{W}_{1}$ & $\mathrm{c}_{1}$ & $a_{11}$ & ....... & $\mathrm{a}_{1 \mathrm{n}}$ \\
\hline$\ldots$ & $\cdots$ & $\ldots \ldots$ & $\ldots \ldots$ & \\
\hline $\mathrm{W}_{\mathrm{m}}$ & $\mathrm{c}_{\mathrm{m}}$ & $a_{\mathrm{m} 1}$ & $\ldots \ldots$ & $a_{m n}$ \\
\hline
\end{tabular}

Table 3. Example of Rules

\begin{tabular}{ccccc}
\hline Rules/Measures & M1 & M2 & M3 & M4 \\
\hline R1: A->B & 0.9 & 0.8 & 0.8 & 0.6 \\
R2: C->D & 0.9 & 0.8 & 0.7 & 0.5 \\
R3 & 0.8 & 0.7 & 0.3 & 0.4 \\
R4 & 0.8 & 0.9 & 0.7 & 0.8 \\
R5 & 0.3 & 0.3 & 0.1 & 0.3 \\
R6 & 0.5 & 0.5 & 0.8 & 0.8 \\
\hline
\end{tabular}

The ELECTRE method is based on the concordance and discordance index defined as follows. We start from the data of the decision table and verify here that the sum of the weights of all criteria equals to 1 .

The concordance index $c_{i k}$ for each and every pair of alternatives $A_{i}$ and $A_{k}(i, k=1, \ldots, m)$ (note that an alternative is not compared to itself) The concordance index lies between 0 and 1 and it is defined as the sum of all the weights for those criteria where the performance score of $A_{\mathrm{i}}$ is least as high as that of $A_{k}$, i.e.

$$
c_{j k}=\sum_{i: a_{i j} \geq a_{i k}} W_{i} \quad j, k=1, \ldots, n \quad j \neq k
$$

Similarly, The computation of the discordance index $d_{j k}$ for each criterion where $A_{k}$ outperforms $A_{j}$ is defined as the maximum of the ratio between the difference in performance level between $A_{k}$ and $A_{j}$ and the maximum difference in score on the criterion concerned between any pair of alternatives. i.e.:

$$
d_{j k}=0 \text { if } a_{i j}>a_{i k}, i=1, \ldots, m,
$$

i.e. the discordance index is zero if $A_{j}$ performs better than $A_{k}$ on all criteria. Otherwise,

$$
\begin{aligned}
d_{j k} & =\max _{i=1, \ldots m} \frac{a_{i k}-a_{i j}}{\max _{j=1, \ldots \ldots n} a_{i j}-\min _{j=1, \ldots \ldots n} a_{i j}} \\
j, k & =1, \ldots, n, j \neq k
\end{aligned}
$$

Next, a global concordance threshold $c^{t}$, and a global discordance threshold $d^{t}$, are chosen to execute the global concordance and discordance tests. The more difficult the threshold values are, the more severe it is to pass the tests (normally, $c=0.7$ and $d=0.3$ [19].

For an outranking relation to be judged as true, both global indices should not infringe their corresponding thresholds. That is $C_{i k} \geq c^{t}$ and $D_{i k} \leq d t$. Once the two tests are completed for all pairs of alternatives, the best alternatives are those that outrank more than being outranked.

By establishing such a relation between each and every pair of alternatives, one can then delete the dominated alternatives and achieve the nondominated solutions.

A partial ranking of an outranking method could not provide the best alternative directly. A subset of propositions can be defined such that at least one member of the subset outranks any proposition, not in the subset. The goal is to make this subset smaller. This subset of propositions can be supposed as a shortlist, within which a good compromise proposition should be found by further considerations or methods.

The application of ELECTRE 1 to select the association rules has some disadvantages; it may discard some association rules contain valuable information.

For example in Table 3 , if we take two rules different semantically $R 1: A \rightarrow B$ and $R 2: C \rightarrow D$. By using ELECTRE method:

$C_{12} \geq c^{t}$ and $d_{12} \leq d^{t}$ imply that $R 1$ outrank $R 2$, and $\mathrm{R} 2$ will never exist in the result, which is not logic because the second rule can contain a valuable information as we can see from its high measures.

\section{Our approach}

In this section, a new method of outranking family of MCDA is presented to solve the selection problem of association rules.

This approach is composed of several steps:

*Determination of the relationship among alternatives: this action consists in defining the association between the alternatives concerning each criterion. The pairwise comparison of the alternatives $A_{i}$ and $A_{k}$ where $\mathrm{I}, \mathrm{k} i, k \in\{1, \ldots . n\}$ and $i \neq k$

$$
\operatorname{Set}\left(A_{i}, A_{k}\right)=\left\{j / a_{i j} \geq a_{k j}\right\}
$$

Where $\operatorname{Set}\left(A_{i}, A_{k}\right)$ is the set of criteria for which the alternatives $a_{i}$ is preferred over $a_{k}$.

*The next step is the transformation of the relationship between alternatives, this step consists 
of determining the sum of difference between the alternatives $a_{i}$ and $a_{k}$ in the set $\operatorname{Set}\left(A_{i}, A_{k}\right)$

$$
M\left(A_{i}, A_{k}\right)=\frac{\sum a_{i j}-a_{k j}}{\max a_{i j}-\min a_{i j}} \forall j \in \operatorname{Set}\left(A_{i}, A_{k}\right) \text { (7) }
$$

This index displays how much the hypothesis $\left(A_{i}\right.$ outclass $A_{k}$ ) is consistent with the reality expressed by the evaluation of alternatives.

We can use an amelioration of this step by normalised the formula

$$
\operatorname{NormalM}\left(A_{i}, A_{k}\right)=\frac{M\left(A_{i}, A_{k}\right)}{\sqrt{\sum M^{2}\left(A_{i}, A_{k}\right)}}
$$

This normalisation restricts $\mathrm{m}^{t}$ to be included in the interval $[0,1]$ which help the choice of the threshold by the users.

*The last step is the filtering the alternatives, this step allows extracting from all start in (alternatives, actions) the set of non-outranked actions.

We define the outranking relation $A_{i} O A_{k}$, this relationship means that $A_{i}$ is at least as good as $A_{k}$ if only if $M\left(A_{i}, A_{k}\right) \geq m^{t}$. Where $m^{t}$ is a predefined threshold.

After introducing the enormous number of generated rules via Association Rules Mining Process, using Apriori[1], Close, Close+ [20]or Charm[21], etc. it may be difficult to extract useful information from them; accordingly, we risk to lose information. In this context, we propose to apply Multi-criteria decision analysis (MCDA): our proposed method to find a good compromise without excluding or privileging any measures, which permits to select the most interesting association rules.

After mining association rules from a transactional database $D$, given $R=\left\{R_{1}, R_{2}, \ldots . R_{n}\right\}$ a set of association rules generated by Apriori, and $M=\left\{M_{1}, M_{2}, \ldots . M_{s}\right\}$ a set of measures to evaluate the rules. We denote by $r\left[M_{j}\right]$ the value of the measure $\mathrm{j}$ for the rule r. We consider a set of measures as attributes and the set of rules as objects. So, we take the set of rules as alternatives and a set of measures as criteria to transform decision table.

Let two association rules $R_{i}, R_{k}$. A true outranking relation of $R_{i} \rightarrow R_{k} \quad$ (also denoted as $R_{i} O R_{k}$ ), implies that $R_{i}$ is preferred to $R_{k}$. We say that an association rule $R_{i}$ outranks another association rule $\mathrm{R}_{\mathrm{k}}$ if only if $M\left(R_{i}, R_{k}\right) \geq m^{t}$, (i.e., the sum of the difference between the two are within a predefined threshold $m^{t}$ ).
We calculate the $M\left(R_{i}, R_{k}\right)$ index for each and every pair of rules $R_{i}$ and $R_{k}(i, k=1, \ldots, N)$ with $\mathrm{N}$ is the number of all rules. To build an outranking relation, the indices should satisfy their correspondent threshold. $M_{i k}>m^{t}$. The preferred association rules are those that outrank more than being outranked, and the selected association rules are the non-outranked.

Considering the example shown in Table 3, using the data set $\mathrm{D}$ and supposing that $M=\left\{M_{1}, M_{2}, M_{3}, M_{4}\right\}$ the first rule " $R 1$ " outrank " $R 3$ " because

$$
M_{13}=\frac{0.9-0.8}{0.9-0.3}+\frac{0.8-0.7}{0.9-0.3}+\frac{0.8-0.3}{0.8-0.1}+\frac{0.6-0.4}{0.8-0.3}=1.44>m^{t}=1 \text {. }
$$

So $R_{l} O R_{3}$.

$$
M_{14}=\frac{0.9-0.8}{0.9-0.3}+\frac{0.8-0.7}{0.8-0.1}=0.3<m^{t}=1
$$

So $R_{l}$ doesn't outrank $R_{4}$

The procedure of the proposed approach for generating the optimized association rules follows four steps. Firstly, we start by the input of a transactional dataset. Then, we apply Apriori algorithm to find the frequent item sets and generate all association rules. The next step is to calculate the interestingness measures to evaluate the set of rules, and finally, we apply MCDA algorithm to select and choose a set of promising association rules through many interestingness measures.

\section{Experiment study}

We evaluate our proposed method in different public domain datasets: mushroom, flare1, flare2, monks1, monks2, monks3, Zoo. These datasets are available online from UCI Machine Learning Repository. Table 4 shows the properties of the datasets that have been used for our experimental studies. Firstly, we generate association rules using APRIORI[1]. Table 5 shows the minimum support taken for each dataset chosen and the number of rules extracted from the different datasets using Apriori algorithm.

Table 4. Characteristics of the used datasets

\begin{tabular}{lcc}
\hline Data set & items & Transactions \\
\hline Mushroom & 22 & 8124 \\
Flare1 & 32 & 323 \\
Flare2 & 32 & 1066 \\
Monks1 & 19 & 432 \\
Monks2 & 19 & 432 \\
Monks3 & 19 & 432 \\
Zoo & 28 & 101 \\
\hline
\end{tabular}


Table 5. Number of AR generated for each dataset

\begin{tabular}{lcc}
\hline Data set & minsup & $\begin{array}{l}\text { Number of rules } \\
\text { generated }\end{array}$ \\
\hline Mushroom & 40 & 2654 \\
Flare1 & 20 & 3468 \\
Flare2 & 20 & 3342 \\
Zoo & 20 & 3564 \\
Monks1 & 5 & 2422 \\
Monks2 & 5 & 2516 \\
Monks3 & 5 & 2554 \\
\hline
\end{tabular}

As explained in the section (2-2), to evaluate association rules, we use a set of interestingness measures. The measures used for the performed test are Support (SUP), Confidence (CONF), Lift, Information Gain (IG), Example \& Counter Example Rate (ECR), Piatetsky Shapiro (PS), Cosinus (COS) and Jacard (JRD). These measures are calculated using the formulas cited in Table 1.

Now we apply our algorithm to select the most interesting association rules using multi-criteria. The threshold $m^{t}$ is chosen empirically and takes as value 0.2 in all experiments.
In Tables 6, the results obtained from our method are compared with results from ELECTRE [10] and k-means [9]. Also giving the corresponding histograms for the table to illustrate the results.

In the first method, ELECTRE method is proposed as a strategy for association rule (AR) mining within datasets. The second method is based on k-means method and uses this procedure in order to mine rules into clusters, and we compare our result to the best cluster.

First, we show through experiments that our approach can significantly reduce the huge number of rules generated from the data sets, in front of the others approach.

These experiments have the advantage to quantify the reduction of the rules introduced by our approach. Therefore, we compare the number of non-dominated rules of our approach to the number of non-dominated rules of ELECTRE and the first cluster of k-means and the total number of association rules (denoted A-R).

Table 6. The obtained results for different datasets

\begin{tabular}{lccccccc}
\hline & Monks1 & Monks2 & Monks3 & Flare1 & Flare2 & Mushroom & Zoo \\
\hline A.R & 2422 & 2516 & 2554 & 3468 & 3342 & 2654 & 3564 \\
ELECTRE & 1253 & 1072 & 332 & 190 & 477 & 318 & 1722 \\
Our approach & 18 & 52 & 19 & 12 & 192 & 415 & 1724 \\
Our approach Normalised & 48 & 60 & 63 & 69 & 192 & 275 & 1172 \\
K-means & 165 & 648 & 233 & 12 & 192 & 437 & 1336 \\
\hline
\end{tabular}

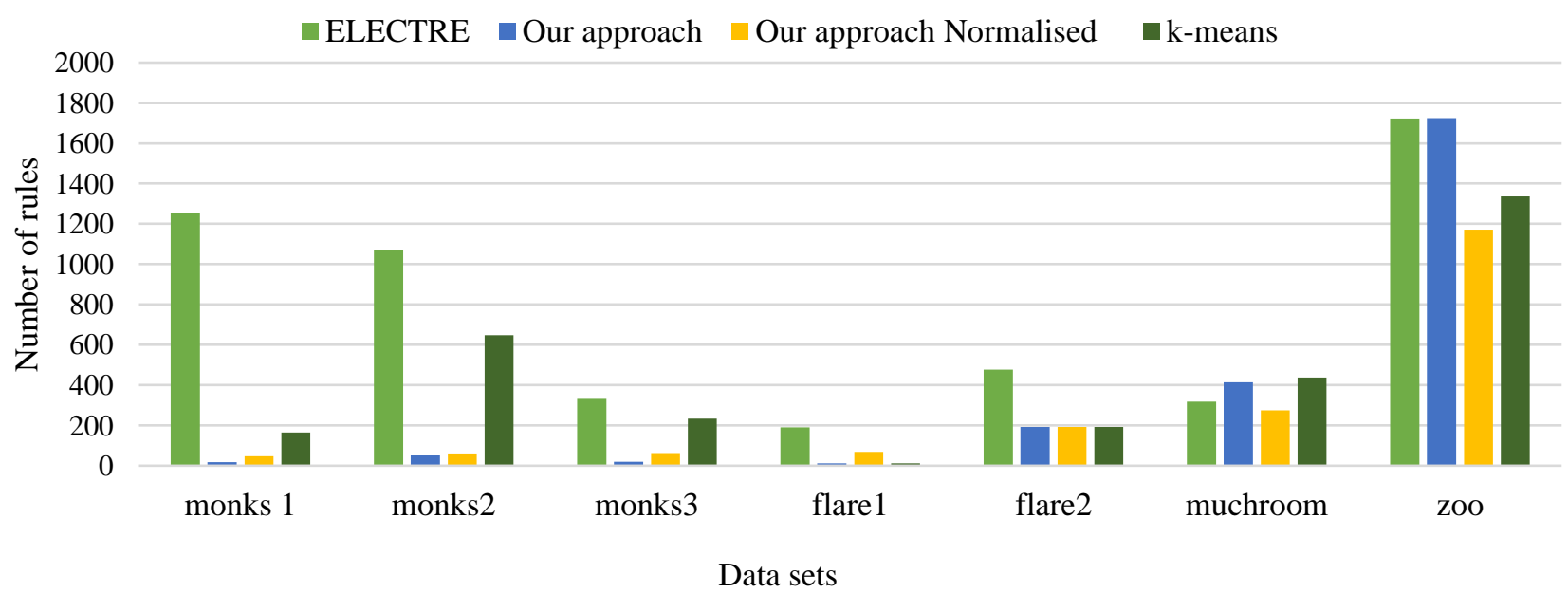

Figure.1 The correspondent histogram of the results 
In order to analyze the performance of our proposed algorithm, we have compared the average value of confidence and the average value of support in each dataset of our method to the ELECTRE method, k-means method, and to the closed algorithm.

The Table 7 and Table 8 show that the proposed method has found rules with high values of confidence and support in the majority of the datasets.

Our observation of the generated rules shows that we have obtained much better results in the case of a number of rules in all chosen datasets. Our results of the confidence measure are much better than previous works. In the support, we had almost the same results. The number of generated rules and their confidence values are more efficient than those of the previous works, and it can be concluded that our generated rules are useful for the users.

Another experiment aims to validate the benefit of our method in front of ELECTRE method, we compute the average of confidence of the set of the intersection between the result of ELECTRE method (E) and the complementary of the result of our approach (CA) and we compare it to the average of confidence of the set of the intersection between the result of our approach (A) and the result of the complementary of the ELECTRE method (CE).

Table 7. The average of confidence for different datasets

\begin{tabular}{llllllll}
\hline & Monks1 & Monks2 & Monks3 & Flare1 & Flare2 & Mushroom & Zoo \\
\hline ELECTRE & 0.313 & 0.294 & 0.42 & 0.6 & 0.84 & 0.92 & 0.48 \\
Our approach & 0.97 & 0.78 & 0.96 & 1 & 0.98 & 0.97 & 0.89 \\
normalized & 0.86 & 0.76 & 0.93 & 0.958 & 0.98 & 0.96 & 0.93 \\
K-means & 0.67 & 0.54 & 0.71 & 1 & 0.98 & 0.79 & 0.92 \\
\hline
\end{tabular}

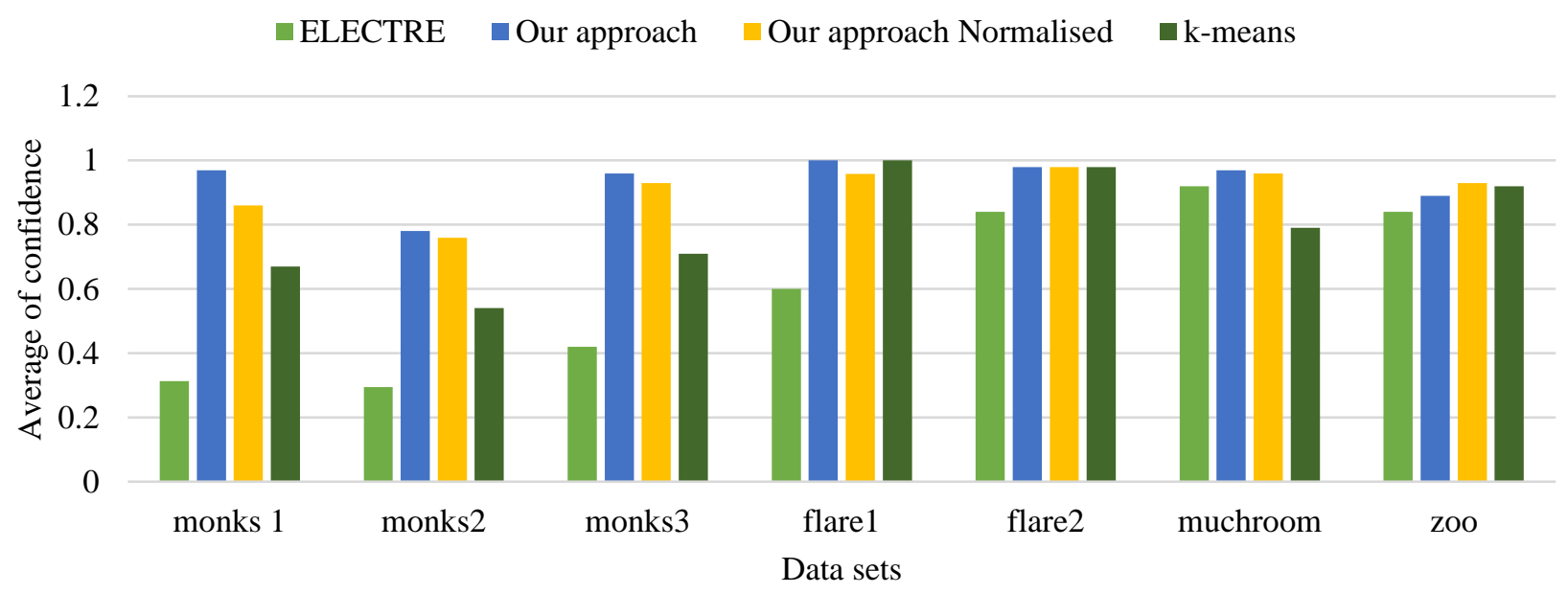

Figure.2 The histogram of the average of confidence

Table 8 . The average of support for different datasets

\begin{tabular}{llllllll}
\hline & Monks1 & Monks2 & Monks3 & Flare1 & Flare2 & Mushroom & Zoo \\
\hline ELECTRE & 0.06 & 0.064 & 0.07 & 0.216 & 0.35 & 0.52 & 0.263 \\
Our approach & 0.11 & 0.099 & 0.131 & 0.201 & 0.242 & 0.517 & 0.277 \\
K-means & 0.07 & 0.09 & 0.08 & 0.2 & 0.24 & 0.42 & 0.27 \\
\hline
\end{tabular}




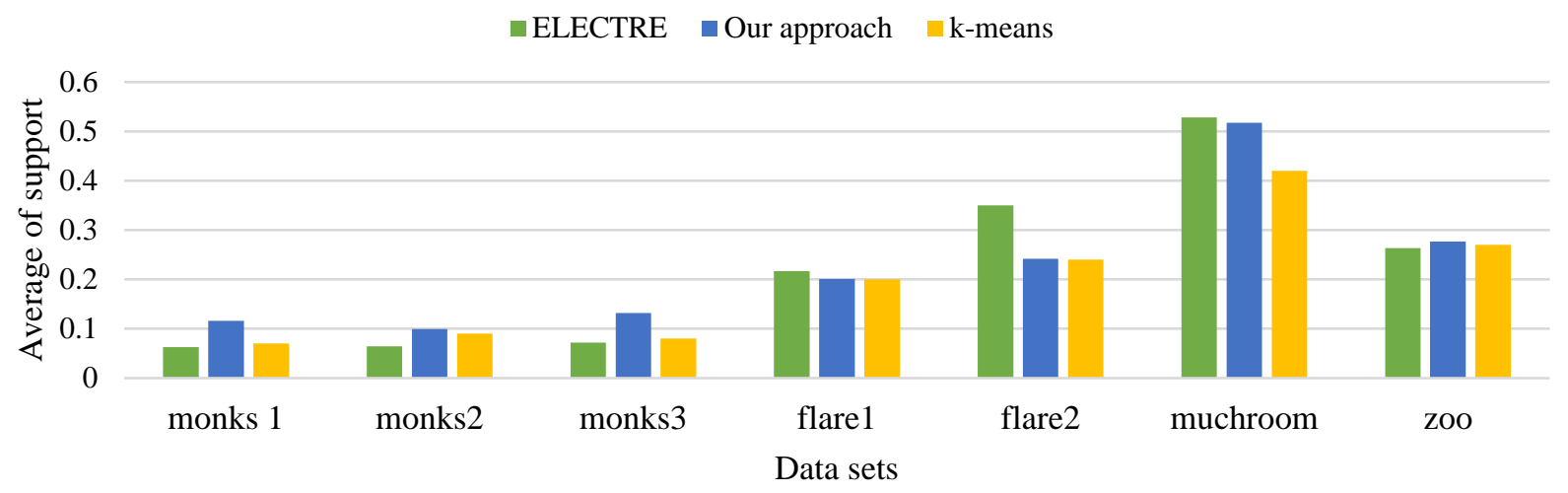

Figure. 3 The histogram of the average of support

Table 9.Comparison of method

\begin{tabular}{lllll}
\hline & Monks2 & Monks3 & Mushroom & Zoo \\
\hline Average of confidence of E inter CA & 0.27 & 0.4 & 0.85 & 0.72 \\
Average of confidence of A inter CE & 0.73 & 0.95 & 0.97 & 0.9 \\
\hline
\end{tabular}

As can be seen in Table 9, the rules left by our method and deleted by ELECTRE are more important than the rules left by ELECTRE method and removed by our approach. The reason why we deduce that our proposed method keep the most interesting association rules and ELECTRE method delete some rules which may contain valuable information.

All those experiments show that our proposed method is better than the existing techniques such as ELECTRE method, which may discard some rules contain a valuable information and have a high value of measures. However, our method keeps the most interesting ones. The reason theoretical of this benefice is due to the proposed new outranking relation which is already explained above.

\section{Conclusion}

In this paper, we present a new multicriteria decision-making method based on ELECTRE method which is able to select the most interesting association rules generated using apriori by consideration a new outranking relation. The main advantage of the proposed method is that it is not hindered by the abundance of measures and it evaluates the association rules using a set of criteria, not only one. In comparison to previous works, the experimental results show that our approach has the ability to reduce a large number of association rules which help the users to take a decision, on the other hand, the selected rules are more significant and interesting with high
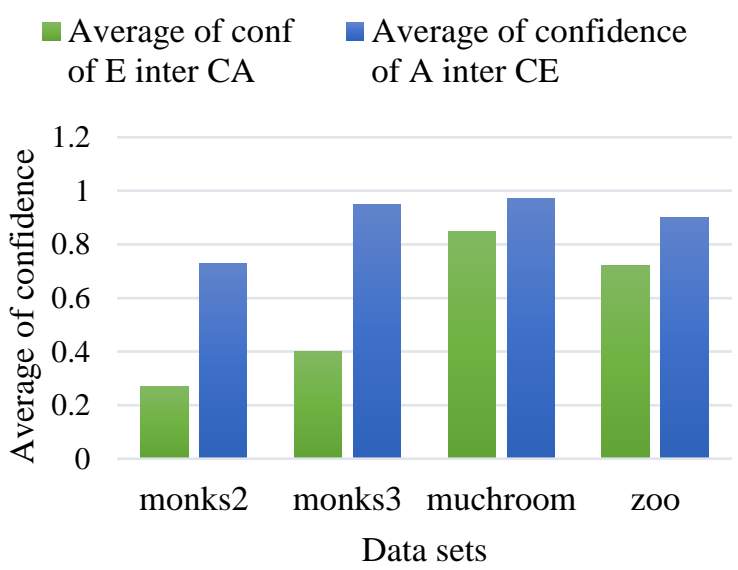

Figure. 4 The histogram of comparison of the method

accuracy and efficiency. As a part of future work, we would like to extend our work to rank all association rules and adding the semantic notion.

\section{References}

[1] R. Agrawal and R. Srikant, "Fast algorithms for mining association rules", In: Proc. Of International Conf. Very Large Data Bases, Santiago, Chile, pp. 487-499, 1994.

[2] A. Silberschatz and A.Tuzhilin, "What Makes Pattens Interesting in Knowledge Discovery Systems", IEEE Transactions on Knowledge and Data Engineering, Vol.8, No.6, pp. 970974, 1996.

[3] H. X. Huynh, F. Guillet, T. Q. Le, and H. Briand, "Ranking objective interestingness 
measures with sensitivity values", $V N U$ Journal of science: Natural Sciences and technology, Vol. 24, pp. 122-132, 2008.

[4] R. Gras, R. Couturier, J. Blanchard, H. Briand, P. Kuntz, and P. Petter, Quelques critères pour une mesure de qualité de règles d'association, In, RNTI-E-1, Cépaduès Editions., pp. 3-32, 2004.

[5] M. Gavrilov, D. Anguelov, P. Indyk, and R. Motwani, "Mining The Stock Market: Which Measure Is Best?", In : proc. Of International Conf. on Knowledge discovery and data mining, Boston, USA, pp. 487-496, 2000.

[6] B. Vaillant, P. Lenca, and S. Lallich, "A clustering of interestingness measures," In: Proc. of International Conference. On Discovery Science, Padova, Italy, pp. 290-297, 2004.

[7] M. C. Chen, "Ranking discovered rules from data mining with multiple criteria by data envelopment analysis", Expert Systems with Applications, Vol.33, No.4, pp.1110-1116, 2007.

[8] M. Toloo, B. Sohrabi, and S. Nalchigar,"A new method for ranking discovered rules from data mining by DEA," Expert Systems with Applications, Vol.36, No. 4, pp. 8503-8508, 2009.

[9] A. Dahbi, M. Mouhir, Y. Ballouki, and T. Gadi, "Classification of association rules based on k-means method," In: Proc. of the 4th IEEE International Colloquium on Information Science and Technology, Tangier-Assilah, Morocco, pp. 300-305, 2016.

[10] A. Dahbi, S.Jabri, Y. Ballouki, and T. Gadi, "Using ELECTRE method to select the interesting association rules with multiple criteria". In: Proc. of 1st International Conference on Computing Wireless and Communication Systems, Settat, Morocco, pp. 80-85, 2016.

[11] L. Cao, D. Luo, and C. Zhang, "Knowledge actionability: satisfying technical and business interestingness", International Journal of Business Intelligence and Data Mining., Vol. 2, No. 4, pp. 496-514, 2007.

[12] P.-N. Tan, V. Kumar, and J. Srivastava, "Selecting the Right Interestingness Measure for Association Patterns," In: Proc. eighth ACM SIGKDD Int. Conf. Knowl. Discov. Data Min, Edmonton, Alberta, Canada, pp. 32-41, 2002.

[13] B. Liu, W. Hsu, S. Chen, and Y. Ma, "Analyzing the subjective interestigness of association rules", IEEE Intelligent Systems and their Applications, Vol.15, no.5, pp. 47$55,2000$.

[14] S. O. Rezende, E. A. Melanda, M. L. Fujimoto, R. A. Sinoara, and V. O. de Carvalho, "Combining data-driven and userdriven evaluation measures to identify interesting rules", Post-Mining of Association Rules: Techniques for Effective Knowledge Extraction, IGI Global, pp. 38-55, 2009.

[15] R. Paul, T. Groza, J. Hunter, and A. Zankl, "Semantic interestingness measures for discovering association rules in the skeletal dysplasia domain", Journal of Biomedical Semantics, Vol.5, No.1, pp.5-8, 2014.

[16] L. Geng and H. J. Hamilton, "Interestingness measures for data mining", ACM Computing Surveys, Vol.38, No.3, pp. 1-32, 2006.

[17] M. Velasquez and P. T. Hester, "An Analysis of Multi-Criteria Decision Making Methods", International Journal of Operations Research, Vol.10, No.2, pp.56-66, 2013.

[18] J. Dyer, Multiple Criteria Decision Analysis: State of the Art Surveys, Vol 78, SpringerVerlag, 2005.

[19] A. S. Milani, A. Shanian, and C. El-Lahham, "Using different ELECTRE methods in strategic planning in the presence of human behavioral resistance", Journal of Applied Mathematics and Decision Sciences, Vol. 2006, pp. 1-19, 2006.

[20] J. Wang, J. Han, and J. Pei, "Closett: Searching for the best strategies for mining frequent closed itemsets", In: Proc. ninth ACM SIGKDD Int. Conf. Knowl. Discov. data Min, Washington, D.C, USA, pp. 236-245, 2003.

[21] M. J. Zaki and C-J. Hsiao, "CHARM: An efficient algorithm for closed association rule mining", In: Proc.of the 2nd SIAM Int. Conf. Data Min., Chicago, USA, pp.457-473, 1999. 